\title{
Long-term Reverse Remodeling by Cardiac Resynchronization Therapy with MultiPoint Pacing: A Feasibility Study of Noninvasive Hemodynamics Guided Device Programming
}

Short Title: Noninvasive Hemodynamics to Guide CRT and MPP Programming

Peter Lercher ${ }^{1}, \mathrm{MD}$; Maurizio Lunati ${ }^{2}, \mathrm{MD}$; Roberto Rordorf ${ }^{3}, \mathrm{MD} ;$ Maurizio Landolina ${ }^{3,4}, \mathrm{MD}$; Nima Badie $^{5}$, PhD; Fujian Qu ${ }^{5}$, DSc; Cyrille Casset ${ }^{5}$, MEng; Kyungmoo Ryu ${ }^{5}$, PhD; Stefano Ghio ${ }^{3}$, MD; Jagmeet P. Singh $^{6}, \mathrm{MD}, \mathrm{PhD}, \mathrm{FHRS}$; Christophe Leclercq ${ }^{7}, \mathrm{MD}, \mathrm{PhD}$

${ }^{1}$ Department of Cardiology, Medical University Graz, Graz, Austria

${ }^{2}$ Cardiac Department, Niguarda Ca' Granda, Granda Hospital, Milan, Italy

${ }^{3}$ Cardiac Department, Policlinico San Matteo, Pavia, Italy

${ }^{4}$ Cardiology Department, Ospedale Maggiore di Crema, Crema, Italy

${ }^{5}$ Abbott, Abbott Park, Illinois, USA

${ }^{6}$ Cardiac Arrhythmia Service, Massachusetts General Hospital, Harvard Medical School, Boston, Massachusetts, USA

${ }^{7}$ Department of Cardiology, University Hospital of Rennes, Rennes, France

Financial Support and Conflicts of Interest:

The study was funded by Abbott. Maurizio Lunati received funding from Medtronic, Abbott, Sorin, Boston Scientific, Biotronik, Boehringer-Ingelheim, and Bayer. Roberto Rordorf received funding from Medtronic, Abbott, and Boston Scientific. Maurizio Landolina received funding from Medtronic. Nima Badie, Fujian Qu, Cyrille Casset, and Kyungmoo Ryu are employees of Abbott. Jagmeet Singh received funding from Abbott, Boston Scientific, Medtronic, Sorin, Cardiolnsight, Respicardia, and Biotronik. Christophe Leclercq received funding from Medtronic, Sorin, Boston Scientific, Biotronik, Bayer, and Abbott.

Word Count (excluding title page): 4,871

Please direct correspondence to:

Christophe Leclercq, MD, PhD

Département de Cardiologie et Maladies Vasculaires

CHU Pontchaillou

2, rue Henri le Guilloux, 35033 Rennes cédex 09, France.

Fax: 33-2-99-28-25-10

E-mail: christophe.leclercq@chu-rennes.fr 


\section{Abstract}

Background: Cardiac resynchronization therapy (CRT) with multipoint left ventricular (LV) pacing (MultiPoint ${ }^{\mathrm{TM}}$ Pacing [MPP]) improves acute hemodynamics and chronic outcomes over conventional biventricular pacing (BiV), though MPP programming questions persist.

Objective: In this multi-center feasibility study, we evaluated the feasibility of using noninvasive systolic blood pressure (SBP) to guide MPP programming, and assessed the chronic 6-month echocardiographic CRT response.

Methods: Patients implanted with MPP-enabled CRT defibrillator (CRT-D) devices underwent noninvasive hemodynamic assessment (finger arterial pressure) during a pacing protocol that included atrial-only pacing and various BiV and MPP configurations. Each configuration was repeated 4 times, alternating with reference, to calculate the SBP difference relative to reference ( $\triangle S B P$ ). CRT configurations with the greatest $\triangle \mathrm{SBP}$ were programmed. An independent core lab analyzed baseline and 6-month echocardiography, with CRT response defined as a 6-month reduction in LV end-systolic volume $(\triangle \mathrm{LVESV}) \geq 15 \%$.

Results: Forty-two patients (71\% male, LV ejection fraction $30.3 \pm 7.5 \%$, QRS duration $161 \pm 19 \mathrm{~ms}, 26 \%$ ischemic) were enrolled in 4 European centers. Relative to atrial-only pacing, the best BiV and best MPP configurations produced significant SBP elevations of $3.1 \pm 4.2(p<0.01)$ and $4.1 \pm 4.1 \mathrm{mmHg}(p<0.01)$, respectively (BiV vs MPP, $p<0.01$ ). Greater SBP elevations were associated with the best MPP compared to the best BiV configurations in 29/37 (78\%) of patients completing the pacing protocol. Of MPPprogrammed patients completing the 6-month follow-up, $23 / 27$ (85\%) were classified as CRT responders $(\Delta \mathrm{LVESV}=31 \pm 20 \%)$.

Conclusion: Acute noninvasive hemodynamics after CRT implant predominantly favored MPP over BiV programming. MPP programming guided by noninvasive hemodynamics resulted in positive LV structural remodeling.

Key Words: Cardiac resynchronization therapy; heart failure; reverse remodeling; noninvasive hemodynamics; Multipoint Pacing; end-systolic volume; finger arterial pressure 


\section{Introduction}

Conventional cardiac resynchronization therapy (CRT) involves simultaneous pacing of the right ventricle (RV) and the left ventricle (LV). A coronary sinus lead, typically in a lateral or posterolateral coronary vein, is placed for LV pacing in addition to RV endocardial lead. Results from randomized, controlled clinical trials have consistently demonstrated that CRT improves cardiac structure and function while significantly reducing the risk of worsening heart failure. ${ }^{1}$ However, up to $40 \%$ of the patients fail to respond to this therapy. ${ }^{2}$

In an effort to improve CRT response, methods to deliver pacing at multiple LV sites have been developed. Although attractive from a physiological perspective, dual-vein multisite LV pacing is hindered by technical complexities and several clinical issues. ${ }^{3-7} \mathrm{~A}$ recently introduced technique to achieve multisite LV pacing without the need for two LV leads makes use of a quadripolar LV lead paired with a pulse generator capable of pacing from two LV electrodes in the same coronary vein (MultiPoint ${ }^{\mathrm{TM}}$ Pacing [MPP] technology, Abbott, Abbott Park, IL).

Initial clinical experience has shown that MPP, compared with conventional BiV pacing, provides acute benefits to $\mathrm{LV} \mathrm{dP} / \mathrm{dt}_{\operatorname{Max}}{ }^{8-10}, \mathrm{LV}$ dyssynchrony ${ }^{11}$, LV peak radial strain ${ }^{12-13}$, and LV electrical activation ${ }^{14}$, and improves long-term CRT response ${ }^{15-17}$. However, questions persist on how to program MPP in a routine clinical setting. Noninvasive methods have the advantage of being convenient to acquire, free from potential complications associated with invasive measurements, and allow multiple measurements to be made to increase the confidence of measurements. In this multi-center feasibility study, we evaluated the feasibility of using noninvasive systolic blood pressure (SBP) to guide MPP programming, and assessed the chronic echocardiographic CRT response at 6 months.

\section{Methods}

This study was conducted at 4 European centers. All patients provided written informed consent, and the study protocol was reviewed and approved by the Human Research Ethics Committee of each participating institution. The patient population included in this study was characterized by NYHA functional class II or III and indicated by ESC/EHRA guidelines to undergo de novo implant of a CRT defibrillator (CRT-D) system. Patients were excluded for any of the following conditions: age <18 years, pregnancy or planned pregnancy within 12 months, persistent or permanent atrial fibrillation, recent myocardial infarction within 40 days, cardiac surgery or coronary revascularization procedure within 3 months or scheduled for such procedure in the following 12 months, recent cerebrovascular accident or transient ischemic attack within 3 months, on intravenous inotropic support in the last 30 days, or either scheduled or considered for heart transplantation over the next 12 months. The study was registered on ClinicalTrials.gov (NCT02064751).

\section{Procedures and Follow-up Visits}

Abbott CRT-D devices with the MPP feature (Quadra Assura MPTM, models 3371-400/QC) and Quartet ${ }^{\circledR}$ quadripolar LV pacing lead were used in this study. Patients underwent device implant according to standard practice. Prior to discharge, each patient underwent noninvasive hemodynamic assessment 
under a study-specific pacing protocol and was programmed to the CRT configuration that yielded the greatest increase in systolic blood pressure. Noninvasive hemodynamic assessment was repeated at the 6-month follow-up visit.

Echocardiographic evaluation was performed at the pre-implant baseline visit and again at 6 months post-implant. LV end-systolic volume (LVESV) and LV ejection fraction (LVEF) were analyzed by a core lab. Patients were considered to be responders to CRT at the 6-month follow-up visit if they demonstrated a reduction in LVESV of at least $15 \%$ relative to baseline ( $\triangle$ LVESV $\geq 15 \%$ ). Clinical evaluations including NYHA functional class, 6 minute hall walk distance (6MWD), and Minnesota living with heart failure questionnaire (MLWHF) were collected at baseline and every follow-up visit.

\section{Selection of Pacing Configurations}

Atrial-only pacing, conventional biventricular pacing (BiV) configurations, and MPP configurations were evaluated at the post-implant hospital discharge (pre-discharge) visit, as shown in Table 1. The cathodeanode combinations that comprised the LV pacing vectors of interest included the 4 quadripolar LV lead electrodes (D1, M2, M3, P4: distal-to-proximal) along with the RV coil. LV pacing vectors with a capture threshold higher than $3.5 \mathrm{~V}$ or presence of phrenic nerve stimulation (PNS) at twice the capture threshold were excluded. The 4 BiV configurations tested included each of the 4 quadripolar LV lead electrodes as cathodes, with the RV coil as anode.

Two MPP vector combinations ("anatomically-defined MPP" and "electrically-defined MPP") were selected for testing. The anatomically-defined MPP configuration was chosen to pace first using a distal electrode as cathode (LV1) and second using a proximal electrode as cathode (LV2) with maximum separation between the two cathodes along the quadripolar lead body. The electrically-defined MPP configuration was selected to pace first using the cathode with the earliest electrical activation (LV1) and second using the cathode with the latest electrical activation (LV2), as measured from RV pacing to LV sensing at each of the 4 quadripolar LV lead electrodes. RV coil was used as the anode of the MPP LV pacing vectors. An additional MPP configuration was tested, using D1-M2 as the LV1 pacing vector and $\mathrm{M} 3-\mathrm{RV}$ coil as the LV2 pacing vector with LV1 $\rightarrow \mathrm{LV} 2$ and LV2 $\rightarrow$ RV delays of 10 ms each.

The device can or an LV electrode was substituted for the RV coil as the anode in BiV or MPP configurations when high capture threshold or PNS was observed.

\section{Pacing Protocol and Noninvasive Hemodynamic Assessment}

Each of the above pacing configurations defined a Test Pacing Intervention (TPI). To minimize the effect of intrinsic blood pressure variation, a fixed pacing configuration was used as the Reference Pacing Intervention (RPI) between all repeated TPIs. In order to minimize abrupt changes in hemodynamics between pacing transitions and better characterize benefit of MPP over BiV pacing, the RPI for each patient was defined as BiV pacing using the distal LV electrode as cathode and RV coil as anode. Each TPI alternated with the RPI four times to yield eight pacing transitions between TPI and RPI. All pacing interventions were delivered for $20 \mathrm{sec}$ at a time, with a pacing constant rate above the patient's 
intrinsic rate, and a fixed atrioventricular (AV) delay (between 100-150ms) to ensure consistent ventricular capture.

Noninvasive hemodynamics were measured using a finger arterial pressure system (Finometer MIDI, Finapres Medical Systems, Netherlands). The relative change in systolic blood pressure ( $\triangle \mathrm{SBP}$ ) was calculated between each TPI and neighboring RPI by comparing the mean SBP 10 seconds immediately before and after a pacing transition. The mean and standard deviation of $\triangle$ SBP from the eight pacing transitions were used to rank each TPI relative to the RPI. The use of $\triangle$ SBP to guide AV delay programming has been previously described by Whinnett et $\mathrm{al}^{18}$.

Prior to hospital discharge, patients were programmed to the BiV or MPP TPI associated with the greatest mean $\triangle$ SBP. If no MPP vector combination was available, the patient was programmed at the discretion of the investigator and withdrawn from the study.

\section{Statistical Analysis}

All measurements were expressed as mean \pm standard deviation. Comparisons of different pacing interventions within each patient were made by using paired $t$ tests. Comparisons of patient subgroups were made using unpaired $t$ tests. $P$ values $<0.05$ were considered significant.

\section{Results}

\section{Study Population}

Forty-five patients scheduled to undergo de novo implantation of a CRT-D device (Quadra Assura MP'M with Quartet ${ }^{\mathrm{TM}}$ LV lead, Abbott) were enrolled in 4 European centers. Of those patients, 42 completed the baseline visit and implant (withdrawn: 1 presented in persistent AF, 1 with baseline LVEF > 35\%, 1 had an unknown paracardiac mass identified by MRI), 39 patients completed the pre-discharge data collection visit (withdrawn: 3 presented high capture thresholds), and 34 patients participated through the 6 month follow-up (withdrawn: 2 exhibited PNS, 2 LV lead dislodgement, 1 patient request). Patients were implanted between May, 2014 and June, 2015. The distributions of the implanted RV and LV lead locations are shown in Figure 1. Pre-implant baseline patient demographics, comorbidities, and HF metrics for all patients with complete 6 month data are shown in Table 2 .

\section{Acute Hemodynamic Evaluation: Pre-discharge}

Noninvasive finger arterial blood pressure measurements at pre-discharge demonstrated a greater acute response to MPP compared to BiV configurations. The BiV and MPP configurations listed in Table 1 were ranked for each patient according to SBP elevation relative to atrial-only pacing. As shown in the top-left panel of Figure 2, the best BiV and best MPP configurations produced significant SBP elevations of $3.2 \pm 4.3(p<0.001)$ and $4.1 \pm 4.2 \mathrm{mmHg}(p<0.001)$, relative to atrial-only pacing, respectively with a significant difference between best MPP and best BiV (MPP-BiV: $0.9 \mathrm{mmHg}, p<0.001$ ). 
Furthermore, the SBP changes elicited by the best BiV and best MPP configurations, relative to atrialonly pacing, were slightly greater for non-ischemic (BiV: $3.5 \pm 4.1$, MPP: $4.4 \pm 4.1 \mathrm{mmHg}$ ) vs. ischemic patients (BiV: $2.0 \pm 4.9$, MPP: $3.0 \pm 4.4 \mathrm{mmHg}$ ), as shown in the middle-left panels ( $\triangle \mathrm{BiV}: 1.5, \triangle \mathrm{MPP}: 1.4$ $\mathrm{mmHg}$ ). Similarly, these changes were also slightly greater for patients with QRS duration > $150 \mathrm{~ms}$ (BiV: 4.1 \pm 4.4 , MPP: $4.9 \pm 4.4$ ) vs. those with QRS duration $\leq 150 \mathrm{~ms}$ (BiV: $1.2 \pm 3.2$, MPP: $2.3 \pm 2.9$ ), as shown in the bottom-left panels ( $\triangle$ BiV: 2.9, $\triangle \mathrm{MPP}: 2.6 \mathrm{mmHg}$ ).

The SBP changes were highly dependent on the specific CRT settings, with a broad range of $\triangle \mathrm{SBP}$ magnitudes observed. Differences in $\triangle \mathrm{SBP}$ between the best and worst settings were $1.6 \pm 1.3 \mathrm{mmHg}$ for BiV configurations and $4.4 \pm 1.9 \mathrm{mmHg}$ for MPP configurations, indicating the importance of MPP setting selection.

Greater acute SBP elevations were associated with the best MPP configuration compared to the best BiV configuration in $31 / 39(79 \%)$ of patients who completed the pacing protocol. Each patient was ultimately programmed to the BiV or MPP configuration associated with the highest acute SBP elevation. Patients programmed to BiV, in comparison to those programmed to MPP, had longer baseline QRS duration ( $169 \pm 29$ vs. $158 \pm 29 \mathrm{~ms}$ ) and less RV-to-LV electrical conduction dyssynchrony ( $13 \pm 12$ vs. $21 \pm 18 \mathrm{~ms}$, measured by the difference in activation times among the $4 \mathrm{LV}$ electrodes during $\mathrm{RV}$ pacing). There was no difference in heart failure etiology between patients programmed to MPP and Biv.

The distribution of pacing electrodes (cathodes) associated with the best BiV and best MPP configurations is provided in Table 3. Although a single cathode was not particularly favored in BiV configurations, the best MPP configurations favored D1 and either P4 or M3 as cathodes. In terms of $\mathrm{LV} 1 \rightarrow \mathrm{LV} 2 \rightarrow \mathrm{RV}$ pacing delays in the best MPP configurations, 18/31 (58\%) included delays $>20 \mathrm{~ms}$, while $13 / 31(42 \%)$ included only delays $\leq 20 \mathrm{~ms}$.

\section{Acute Hemodynamic Evaluation: 6 Months Post-implant}

Repeated noninvasive finger blood pressure measurements 6 months post-implant revealed a sustained acute benefit of MPP over BiV configurations, as shown in the right panels of Figure 2. Relative to atrialonly pacing, the best BiV and best MPP configurations at 6 months post-implant produced significant SBP elevations of $2.9 \pm 5.1(p<0.01)$ and $3.9 \pm 4.7 \mathrm{mmHg}(p<0.01)$, respectively (MPP-BiV: $1.0 \mathrm{mmHg}$, $\mathrm{p}<0.01)$.

Similar to pre-discharge measurements, the SBP changes elicited by the best BiV and best MPP configurations were slightly greater for non-ischemic (BiV: $3.5 \pm 5.7$, MPP: $4.8 \pm 5.1 \mathrm{mmHg}$ ) vs. ischemic patients (BiV: $1.7 \pm 3.2$, MPP: $2.1 \pm 2.9 \mathrm{mmHg}$ ), as shown in the middle-right panels of Figure 2 ( $\triangle \mathrm{BiV}: 1.8$, $\triangle M P P: 2.7 \mathrm{mmHg}$ ). These changes were also slightly greater for patients with QRS duration $>150 \mathrm{~ms}$ (BiV: $3.7 \pm 5.8$, MPP: $4.7 \pm 5.2$ ) vs. those with QRS duration $\leq 150 \mathrm{~ms}$ (BiV: $1.0 \pm 1.7$, MPP: $2.1 \pm 2.2$ ), as shown in the bottom-right panels ( $\triangle \mathrm{BiV}: 2.7, \triangle \mathrm{MPP}: 2.6 \mathrm{mmHg}$ ).

Also consistent with pre-discharge measurements, the best and worst BiV and MPP configurations resulted in a broad range of SBP changes. Differences in $\triangle S B P$ between the best and worst settings 6 
months post-implant were $2.3 \pm 2.4 \mathrm{mmHg}$ for BiV configurations and $5.6 \pm 2.5 \mathrm{mmHg}$ for MPP configurations.

\section{Chronic Outcomes at 6 and 12 Months Post-implant}

In terms of $\triangle \mathrm{LVESV} \geq 15 \%$ at 6 -months relative to pre-implant, $4 / 5$ BiV patients and $24 / 29$ MPP patients were classified as CRT responders, for a total response rate of 28/34 (82.4\%), shown in Figure 3 . All 6 non-responders were male, 2 had ischemic cardiomyopathy, 4 had LV leads in lateral and 2 in posterolateral vein branches, and RV leads were septal in 2 patients and apical in 4 patients. The single BiV non-responder was programed to BiV simultaneous pacing using the D1 LV electrode. Among the 5 MPP non-responders, 3 were programmed with D1 and P4 LV electrodes, 1 with D1 and M3, and 1 with M2 and P4. CRT responder rate was $9 / 11(81.8 \%)$ in ischemic patients and 19/23 (82.6\%) in nonischemic patients.

Chronic 6-month LV remodeling outcomes assessed using echocardiography are shown in Figure 4. CRT responders and non-responders demonstrated relative LVESV changes of $36.3 \pm 13.0 \%$ and $0.9 \pm 14.6 \%$, respectively, with a collective $\triangle$ LVESV of $30.1 \pm 18.9 \%$. In addition, CRT responders and non-responders demonstrated absolute elevations in LV ejection fraction of $11.3 \pm 5.3 \%$ and $9.0 \pm 6.6 \%$, respectively, with a collective $\triangle$ LVEF of $10.9 \pm 5.6 \%$. MPP-programmed responder patients, specifically, experienced a relative LVESV change of $37.0 \pm 13.6 \%$ and an absolute LVEF elevation of $12.0 \pm 5.2 \%$. $\triangle$ LVESV was $31.8 \pm 15.0 \%$ in ischemic patients and $29.2 \pm 20.8 \%$ in non-ischemic patients. $\triangle$ LVEF was $11.7 \pm 6.1 \%$ in ischemic patients and $10.5 \pm 5.3 \%$ in non-ischemic patients.

As shown in Figure 5, clinical assessment at 6 months showed reduced NYHA functional class ($0.53 \pm 0.66)$, improved quality of life (MLWHF score, $-16.7 \pm 22.5)$, and improved 6MWD $(+37.6 \pm 91.7$ meters) from baseline in all patients. No significant differences between responders and non-responders were observed.

The 6 CRT non-responders at 6 months underwent 9- and 12-month follow-up visits. Devices in these patients were not reprogrammed at 6 months. At 12 months, 2/6 non-responders became responders, $3 / 6$ remained non-responders but showed ESV improvement, and 1 was unknown due to echocardiography errors.

\section{Discussion}

CRT device programming is important in improving patient outcomes. Routine optimization techniques have included echocardiography, device-based algorithms, and other noninvasive techniques, such as blood pressure measurements. To our knowledge, this is the first multi-center study to prospectively evaluate the feasibility of using noninvasive systolic blood pressure measurements to guide MPP programming and subsequently assess the chronic CRT response.

The main findings are (1) the acute noninvasive hemodynamics after CRT implant predominantly favored MPP over BiV programming, and (2) MPP programming guided by systolic blood pressure assessments resulted in positive LV structural remodeling at 6 months post device implant. 


\section{Acute and Chronic Hemodynamics Post-implant}

During the acute hemodynamic assessments after device implant, the best BiV and best MPP configurations (i.e., those yielding the greatest blood pressure elevation) produced significant SBP elevations relative to atrial-only pacing. Greater SBP elevations were associated with the best MPP setting in $79 \%$ of the patients. The evaluated MPP pacing configurations showed a wider range of hemodynamic variation, suggesting acute optimization and individualized programming should definitely be considered to maximize the benefit of MPP. Fortunately, such individualization can be achieved efficiently by noninvasive hemodynamic measurements. The repeated hemodynamic assessment at the 6-month follow-up visit showed a sustained benefit of MPP over BiV configurations.

The achieved SBP elevation in acute optimization protocol was quite small in amplitude and difficult to speculate the clinical implications of the magnitude of response. In our opinion, it is probably more important to compare the SBP elevations by the tested CRT configurations and select the optimal setting. In another word, use it as a metric to identify the CRT setting that results in the highest acute change in SBP rather than link the magnitude of SBP change to long term CRT response.

\section{BiV vs. MPP}

In this study, devices were predominantly programmed to MPP due to superior acute hemodynamics. The 6-month echocardiographic and clinical results in these BiV patients were almost as good as MPP patients. Although statistical significance was not achieved due to sample size limitations, patients programmed to BiV had longer baseline QRS durations and less RV-to-LV conduction dyssynchrony compared to patients programmed to MPP. This observation further underscores the importance of patient-individualized CRT optimization and selective use of MPP in different clinical scenarios.

\section{Importance of Repeated Measurements}

Hemodynamic measurements used to compare CRT configurations are susceptible to confounding factors, such as respiration and baseline signal drift. Previous validation studies ${ }^{19-20}$ have shown that repeated comparisons of test and reference interventions can reduce the impact of confounding factors, allowing the specific effects of the test interventions to be distinguished. Similarly, this study employed a pacing protocol that included four repeated alternations between each TPI and the fixed RPI, resulting in eight replicates of pacing transitions. Although this pacing protocol may seem exhaustive, a recent report showed proof of concept of a simple, fully-automated photoplethysmographic method for AV optimization $^{21}$. Such a system may be applied to automate the pacing protocol used in this study to guide MPP programming.

\section{Dependence of CRT Response on HF Etiology}

In this study, we observed similar improvement in acute noninvasive hemodynamics and long-term reverse remodeling regardless of $\mathrm{HF}$ etiology and QRS duration (greater or less than $150 \mathrm{~ms}$ ). It is well known that HF etiology and lack of dyssynchrony are independent predictors of non-response to conventional CRT. ${ }^{22-23}$ In the CARE-HF and MADIT-CRT study, reverse remodeling was more pronounced 
in the non-ischemic population without a significant impact on clinical outcomes. ${ }^{24-25}$ Conceivably, MPP may provide more benefit in patients with ischemia and heterogeneous patterns of dyssynchrony by providing better synchronization of viable LV tissue and normalizing electrical propagation patterns around the scar tissue. Our observations highlight the potential of further studies to elucidate the impact of MPP with respect to LV scar presence and scar distribution in HF patients.

\section{Chronic Response of MPP Programming Guided by Noninvasive Hemodynamics}

In this study, the CRT response rate and super-response rate (defined by $\triangle \mathrm{LVESV} \geq 30 \%$ ) at 6 months were $82.4 \%$ and $50 \%$, respectively. This high response rate is consistent with previous single-center studies in which acute invasive hemodynamic assessments have been used to guide MPP programming. In a previous study by Pappone et $\mathrm{al}^{15}, \mathrm{CRT}$ response rate and super-response rate based on clinical response at 12 months was $76 \%$ and $33 \%$ in the MPP group, respectively (vs. $57 \%$ and $14 \%$ in the BiV group). Another study by Zanon et $\mathrm{al}^{16}$ reported that CRT response at nearly 1 year post-implant was 90\% when LV pacing site optimization was used with MPP. The results from the current study demonstrate that superior chronic response can be achieved by MPP programming guided by systolic blood pressure measurement using a noninvasive system.

\section{Limitations}

With MPP, the additional LV pacing vector and programmable LV-to-LV delay result in many additional programming options. All of these options were not tested in this study due to the time limitations of an acute pacing protocol. Instead, we prioritized the options based on "anatomically-guided" and "electrically-guided" MPP vector selection methods, both of which are easy to understand and straightforward for clinical practice. In each individual patient, a single AV delay was used in all pacing interventions. Furthermore, BiV pacing interventions were performed with simultaneous BiV pacing, as it is considered standard programming for CRT in routine clinical practice. Whether the benefits of MPP over BiV persist across the physiological range of $\mathrm{AV}$ and interventricular delays remains to be addressed. Another limitation of this feasibility study was the small sample size and relatively high dropout rate for final analysis, which should be supplemented by larger-scale clinical studies.

\section{Conclusion}

This study showed that acute noninvasive hemodynamic evaluation after CRT implant predominantly favored MPP over BiV programming. In addition, MPP programming guided by noninvasive hemodynamics, specifically systolic blood pressure, resulted in positive LV structural remodeling and improved chronic outcomes. These findings have important clinical implications for device programming and CRT optimization using MPP to maximize patient outcomes.

\section{References}


(1) Abraham WT, Hayes DL. Cardiac resynchronization therapy for heart failure. Circulation 2003;108:2596-2603.

(2) Fornwalt BK, Sprague WW, BeDell P, Suever JD, Gerritse B, Merlino JD, Fyfe DA, León AR, Oshinski JN. Agreement is poor among current criteria used to define response to cardiac resynchronization therapy. Circulation 2010;121:1985-1991.

(3) Leclercq C, Gadler F, Kranig W, Ellery S, Gras D, Lazarus A, Clémenty J, Boulogne E, Daubert JC; TRIP-HF (Triple Resynchronization In Paced Heart Failure Patients) Study Group. A randomized comparison of triple-site versus dual-site ventricular stimulation in patients with congestive heart failure. J Am Coll Cardiol 2008;51:1455-1462.

(4) Lenarczyk R, Kowalski O, Kukulski T, Pruszkowska-Skrzep P, Sokal A, Szulik M, Zielińska T, Kowalczyk J, Pluta S, Sredniawa B, Musialik-Łydka A, Kalarus Z. Mid-term outcomes of triple-site vs. conventional cardiac resynchronization therapy: a preliminary study. Int J Cardiol 2009;133:87-94.

(5) Lenarczyk R, Kowalski O, Sredniawa B, Pruszkowska-Skrzep P, Mazurek M, Jędrzejczyk-Patej E, Woźniak A, Pluta S, Głowacki J, Kalarus Z. Implantation feasibility, procedure-related adverse events and lead performance during 1-year follow-up in patients undergoing triple-site cardiac resynchronization therapy: a substudy of TRUST CRT randomized trial. J Cardiovasc Electrophysiol 2012;23:1228-1236.

(6) Rogers DP, Lambiase PD, Lowe MD, Chow AW. A randomized double-blind crossover trial of triventricular versus biventricular pacing in heart failure. Eur J Heart Fail 2012;14:495-505.

(7) Anselme F, Bordachar P, Pasquié JL, Klug D, Leclercq C, Milhem A, Alonso C, Deharo JC, Gras D, Probst V, Piot O, Savouré A. Safety, feasibility, and outcome results of cardiac resynchronization with triple-site ventricular stimulation compared to conventional cardiac resynchronization. Heart Rhythm 2016;13:183-189.

(8) Thibault B, Dubuc M, Khairy P, Guerra PG, Macle L, Rivard L, Roy D, Talajic M, Karst E, Ryu K, Paiement $P$, Farazi TG. Acute haemodynamic comparison of multisite and biventricular pacing with a quadripolar left ventricular lead. Europace 2013;15:984-991.

(9) Pappone C, Ćalović Ž, Vicedomini G, et al. Multipoint left ventricular pacing improves acute hemodynamic response assessed with pressure-volume loops in cardiac resynchronization therapy patients. Heart Rhythm 2014;11:394-401.

(10) Zanon F, Baracca E, Pastore G, et al. Multipoint pacing by a left ventricular quadripolar lead improves the acute hemodynamic response to CRT compared with conventional biventricular pacing at any site. Heart Rhythm 2015;12:975-981.

(11) Rinaldi CA, Kranig W, Leclercq C, et al. Acute effects of multisite left ventricular pacing on mechanical dyssynchrony in patients receiving cardiac resynchronization therapy.J Card Fail 2013;19:731-738. 
(12) Rinaldi CA, Leclercq C, Kranig W, et al. Improvement in acute contractility and hemodynamics with multipoint pacing via a left ventricular quadripolar pacing lead. J Interv Card Electrophysiol 2014;40:75-80.

(13) Osca J, Alonso P, Cano O, Andrés A, Miro V, Tello MJ, Olagüe J, Martínez L, Salvador A. The use of multisite left ventricular pacing via quadripolar lead improves acute haemodynamics and mechanical dyssynchrony assessed by radial strain speckle tracking: initial results. Europace 2016;18:560-567.

(14) Menardi E, Ballari GP, Goletto C, Rossetti G, Vado A. Characterization of ventricular activation pattern and acute hemodynamics during multipoint left ventricular pacing. Heart Rhythm 2015;12:1762-1769.

(15) Pappone C, Ćalović Ž, Vicedomini G, et al. Improving cardiac resynchronization therapy response with multipoint left ventricular pacing: Twelve-month follow-up study. Heart Rhythm 2015;12:1250-1258.

(16) Zanon F, Marcantoni L, Baracca E, et al. Optimization of left ventricular pacing site plus multipoint pacing improves remodeling and clinical response to cardiac resynchronization therapy at 1 year. Heart Rhythm 2016;13:1644-1651.

(17) Forleo GB, Santini L, Giammaria M, et al. Multipoint pacing via a quadripolar left-ventricular lead: preliminary results from the Italian registry on multipoint left-ventricular pacing in cardiac resynchronization therapy (IRON-MPP). Europace 2017;19:1170-1177.

(18) Whinnett ZI, Davies JE, Willson K, Chow AW, Foale RA, Davies DW, Hughes AD, Francis DP, Mayet J. Determination of optimal atrioventricular delay for cardiac resynchronization therapy using acute non-invasive blood pressure. Europace 2006;8:358-366.

(19) Whinnett ZI, Davies JE, Willson K, Manisty CH, Chow AW, Foale RA, Davies DW, Hughes AD, Mayet J, Francis DP. Haemodynamic effects of changes in atrioventricular and interventricular delay in cardiac resynchronisation therapy show a consistent pattern: analysis of shape, magnitude and relative importance of atrioventricular and interventricular delay. Heart 2006;92:1628-1634.

(20) Thibault B, Dubuc $M$, Karst $E$, et al. Design of an acute $\mathrm{dP} / \mathrm{dt}$ hemodynamic measurement protocol to isolate cardiac effect of pacing. J Card Fail 2014;20:365-372.

(21) Kyriacou A, Pabari PA, Whinnett ZI, Arri S, Willson K, Baruah R, Stegemann B, Mayet J, Kanagaratnam $P$, Hughes AD, Francis DP. Fully automatable, reproducible, noninvasive simple plethysmographic optimization: proof of concept and potential for implantability. Pacing Clin Electrophysiol 2012;35:948-960.

(22) Rickard J, Michtalik H, Sharma R, Berger Z, lyoha E, Green AR, Haq N, Robinson KA. Predictors of response to cardiac resynchronization therapy: A systematic review. Int J Cardiol 2016;225:345352.

(23) Brignole M, Auricchio A, Baron-Esquivias G, et al. 2013 ESC Guidelines on cardiac pacing and cardiac resynchronization therapy: the Task Force on cardiac pacing and resynchronization 
therapy of the European Society of Cardiology (ESC). Developed in collaboration with the European Heart Rhythm Association (EHRA). Eur Heart J 2013;34:2281-2329.

(24) Ghio S, Freemantle N, Scelsi L, Serio A, Magrini G, Pasotti M, Shankar A, Cleland JG, Tavazzi L. Long term ventricular reverse remodelling with cardiac resynchronization therapy: results from the CARE-HF trial. Eur J Heart Fail 2009;11: 480-488.

(25) Hsu JC, Solomon SD, Bourgoun M, McNitt S, Goldenberg I, Klein H, Moss AJ, Foster E; MADIT-CRT Executive Committee. Predictors of super-response to cardiac resynchronization therapy and associated improvement in clinical outcome: the MADIT-CRT (multicenter automatic defibrillator implantation trial with cardiac resynchronization therapy) study. J Am Coll Cardiol 2012;59:2366-2373. 


\section{Tables}

Table 1: Pacing configurations tested.

The LV-LV-RV timing for MPP configurations is displayed as "vector ${ }_{1}-\left(\right.$ delay $\left._{1-2}\right) \rightarrow$ vector $_{2}-\left(\right.$ delay $\left._{2-3}\right) \rightarrow$ vector $_{3}$."

\begin{tabular}{|c|c|c|}
\hline CRT Setting & LV Pacing Vectors & Timing \\
\hline Atrial-only & N/A & N/A \\
\hline \multirow{4}{*}{ BiV } & Cathode $=$ D1 & Simultaneous RV + LV \\
\cline { 2 - 3 } & Cathode $=\mathrm{M} 2$ & Simultaneous RV $+\mathrm{LV}$ \\
\cline { 2 - 3 } & Cathode $=\mathrm{M} 3$ & Simultaneous RV $+\mathrm{LV}$ \\
\cline { 2 - 3 } & Cathode $=$ P4 & Simultaneous RV $+\mathrm{LV}$ \\
\hline \multirow{5}{*}{ MPP } & Anatomically-defined & $\mathrm{LV} 1-(5 \mathrm{~ms}) \rightarrow \mathrm{LV} 2-(5 \mathrm{~ms}) \rightarrow \mathrm{RV}$ \\
\cline { 2 - 3 } & Anatomically-defined & $\mathrm{LV} 1-(20 \mathrm{~ms}) \rightarrow \mathrm{LV} 2-(5 \mathrm{~ms}) \rightarrow \mathrm{RV}$ \\
\cline { 2 - 3 } & Anatomically-defined & $\mathrm{LV} 1-(40 \mathrm{~ms}) \rightarrow \mathrm{LV} 2-(5 \mathrm{~ms}) \rightarrow \mathrm{RV}$ \\
\cline { 2 - 3 } & Anatomically-defined & $\mathrm{LV} 1-(5 \mathrm{~ms}) \rightarrow \mathrm{LV} 2-(20 \mathrm{~ms}) \rightarrow \mathrm{RV}$ \\
\cline { 2 - 3 } & Anatomically-defined & $\mathrm{LV} 1-(5 \mathrm{~ms}) \rightarrow \mathrm{LV} 2-(40 \mathrm{~ms}) \rightarrow \mathrm{RV}$ \\
\cline { 2 - 3 } & Electrically-defined & $\mathrm{LV} 1-(5 \mathrm{~ms}) \rightarrow \mathrm{LV} 2-(5 \mathrm{~ms}) \rightarrow \mathrm{RV}$ \\
\cline { 2 - 3 } & Electrically-defined & $\mathrm{LV} 1-(20 \mathrm{~ms}) \rightarrow \mathrm{LV} 2-(5 \mathrm{~ms}) \rightarrow \mathrm{RV}$ \\
\cline { 2 - 3 } & Electrically-defined & $\mathrm{LV} 1-(40 \mathrm{~ms}) \rightarrow \mathrm{LV} 2-(5 \mathrm{~ms}) \rightarrow \mathrm{RV}$ \\
\cline { 2 - 3 } & Additional & $\mathrm{LV} 1-(10 \mathrm{~ms}) \rightarrow \mathrm{LV} 2-(10 \mathrm{~ms}) \rightarrow \mathrm{RV}$ \\
\hline
\end{tabular}

Table 2: Baseline patient characteristics.

\begin{tabular}{|l|c|c|}
\hline \multicolumn{1}{|c|}{ Characteristic } & $\begin{array}{c}\text { Patients completed } \\
\text { Implant } \\
\text { (N = 42) }\end{array}$ & $\begin{array}{c}\text { Patients completed } \\
\text { 6 Month Follow-up } \\
\text { (N = 34) }\end{array}$ \\
\hline Age (yr) & $63 \pm 11$ & $63 \pm 11$ \\
\hline Gender (\% male) & $74 \%$ & $76 \%$ \\
\hline Baseline Ischemic Etiology & $74 \%$ non-ischemic & $68 \%$ non-ischemic \\
\hline NYHA Functional Class & $2.3 \pm 0.5$ & $2.4 \pm 0.5$ \\
\hline QRS Duration (ms) & $160 \pm 20$ & $161 \pm 20$ \\
\hline 6 min Walk Test Distance (m) & $409 \pm 126$ & $408 \pm 134$ \\
\hline LV Ejection Fraction (\%) & $30 \pm 7$ & $30 \pm 7$ \\
\hline LV End-systolic Volume (mL) & $183 \pm 85$ & $188 \pm 87$ \\
\hline LV End-diastolic Volume (mL) & $256 \pm 97$ & $261 \pm 98$ \\
\hline Medication (\% of patients) & $100 \%$ & $100 \%$ \\
ACE Inhibitors / ARBs & $93 \%$ & $94 \%$ \\
Beta Blockers & $90 \%$ & $88 \%$ \\
Diuretics & $50 \%$ & $50 \%$ \\
Antiplatelets & $29 \%$ & $29 \%$ \\
Anticoagulants & $24 \%$ & $24 \%$ \\
Antiarrhythmics Class III & & \\
\hline
\end{tabular}


Table 3: Pacing electrodes associated with greatest $\Delta$ SBP.

\begin{tabular}{|c|c|c|c|}
\hline BiV Cathode & $\begin{array}{l}\text { \# Patients } \\
\text { ( } \mathbf{N}=\mathbf{8} \text { BiV) }\end{array}$ & $\begin{array}{l}\text { MPP Cathode } \\
\text { Combination } \\
\text { (LV1/LV2) }\end{array}$ & $\begin{array}{c}\text { \# Patients } \\
\text { (N = } 31 \text { MPP) }\end{array}$ \\
\hline D1 & 2 & D1/P4 & 10 \\
\hline M2 & 3 & D1/M3 & 7 \\
\hline M3 & 1 & D1/M2 & 2 \\
\hline P4 & 2 & P4/D1 & 3 \\
\hline & & M3/D1 & 3 \\
\hline & & M2/D1 & 2 \\
\hline & & M2/P4 & 2 \\
\hline & & $\mathrm{P} 4 / \mathrm{M} 3$ & 1 \\
\hline & & $\mathrm{M} 2 / \mathrm{M} 3$ & 1 \\
\hline
\end{tabular}

\section{Figure Legends}

Figure 1. Distributions of implanted RV (left) and LV (center, right) lead locations, shown by percent of patients.

Figure 2: Acute hemodynamic assessment at Pre-discharge (left) and 6-month (right) visits. Change in noninvasive systolic blood pressure, relative to atrial-only pacing, shown for the BiV and MPP settings of each patient that elicited the "worst" (smallest change) and "best" (greatest change) acute response. Top panel shows all patients; middle panels group patients by heart failure etiology; bottom panels group patients by QRS duration.

Figure 3: Distribution of 6-month response across all 34 patients. Left pie-chart shows proportion of responders $(\triangle L V E S V \geq 15 \%)$ vs. non-responders. Right pie-chart further distinguishes super-responders $(\triangle L V E S V \geq 30 \%)$ and negative non-responders ( $\triangle$ LVESV $<0 \%$ ).

Figure 4: 6-month LV remodeling outcomes. Mean LVESV and LVEF response of all patients, responder patients $(\triangle L V E S V \geq 15 \%)$, and non-responder patients ( $\triangle L V E S V<15 \%)$. Responder and super-responder $(\Delta L V E S V \geq 30 \%)$ thresholds are indicated as dashed lines. Response for each patient is also shown: super-responder (upward triangle), responder (square), and non-responder (downward triangle).

Figure 5: Clinical parameters at baseline visit and 6-month follow-up. After 6 months, patients had significantly improved NYHA functional class, MLWHFQ score, and 6 minute walk distance $\left({ }^{*} p<0.05\right)$. 
Figure 1

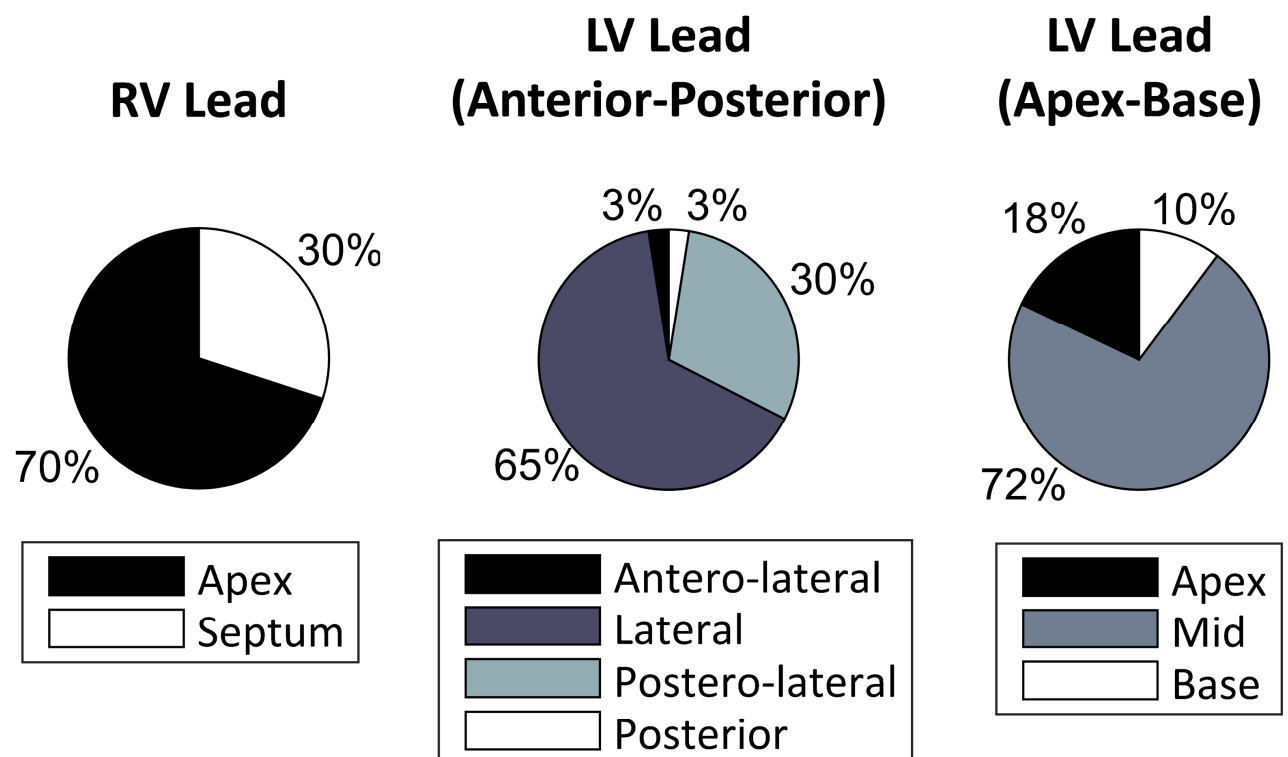




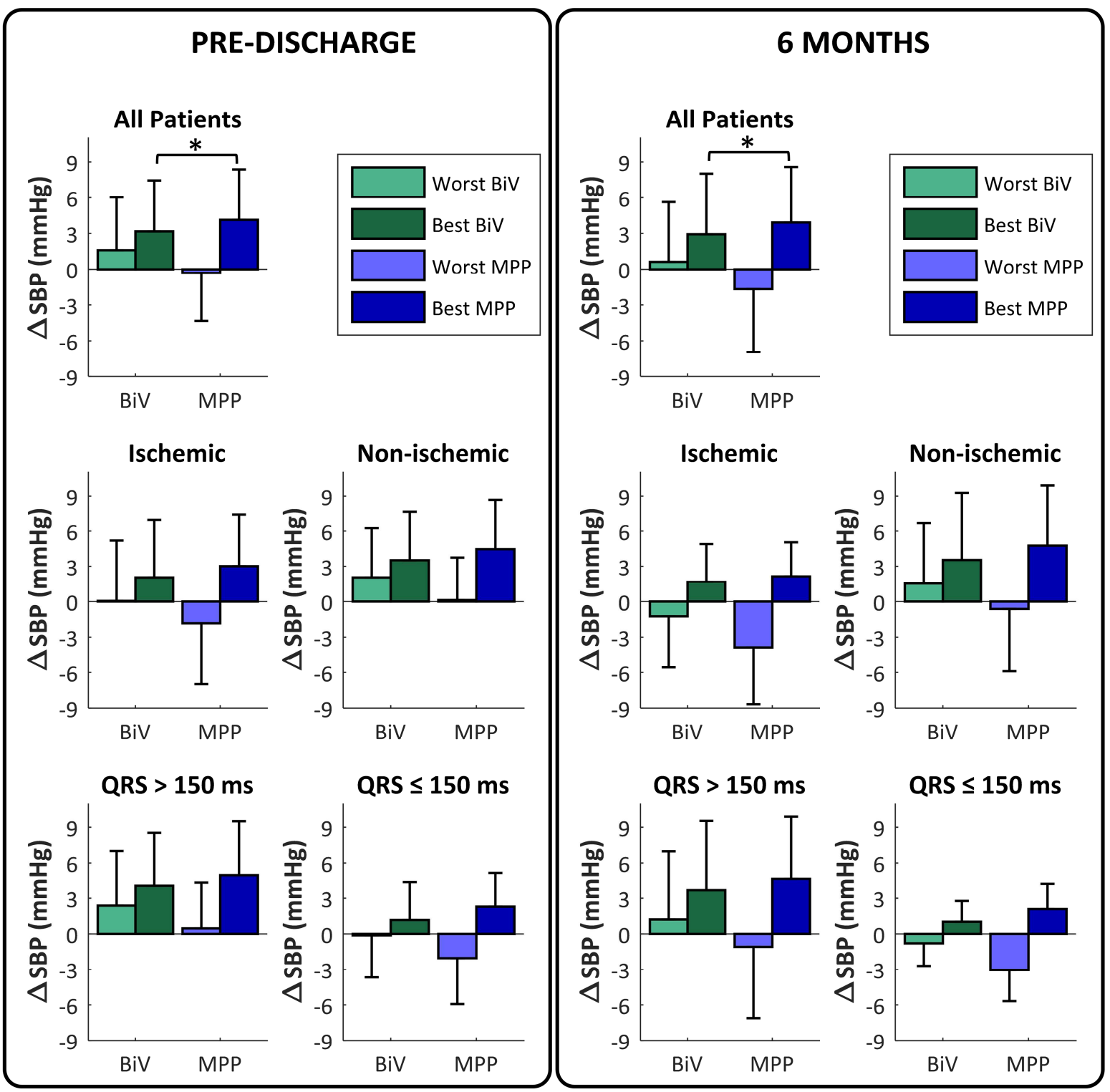


Non-responders

$\Delta$ LVESV $<15 \%$

$17.6 \%$

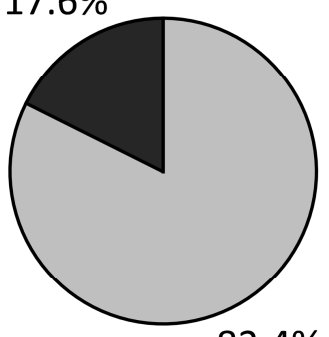

$82.4 \%$

Responders

$\Delta$ LVESV $\geq 15 \%$

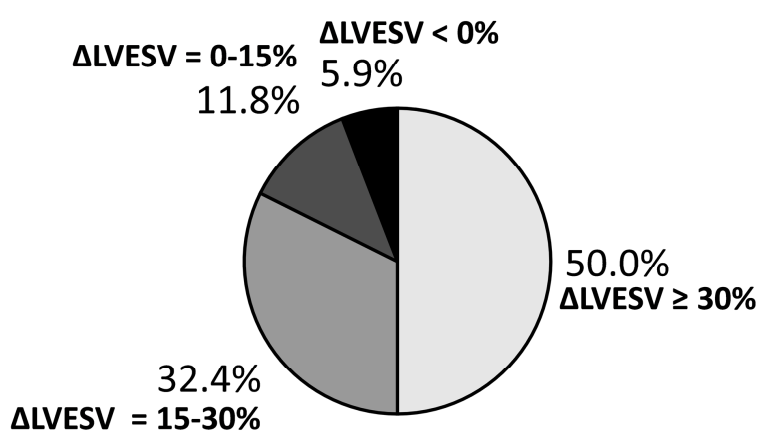


Figure 4
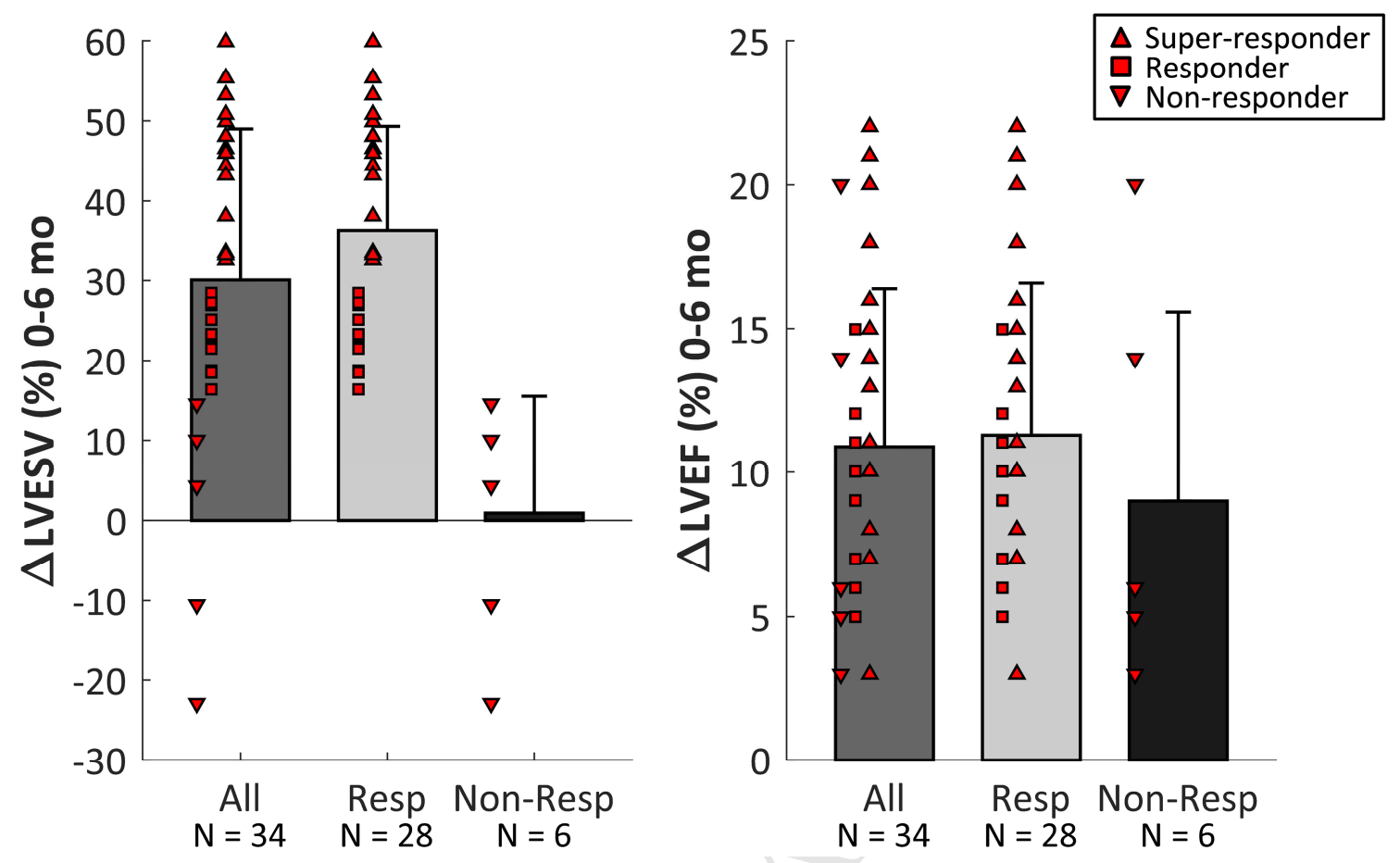
Figure 5
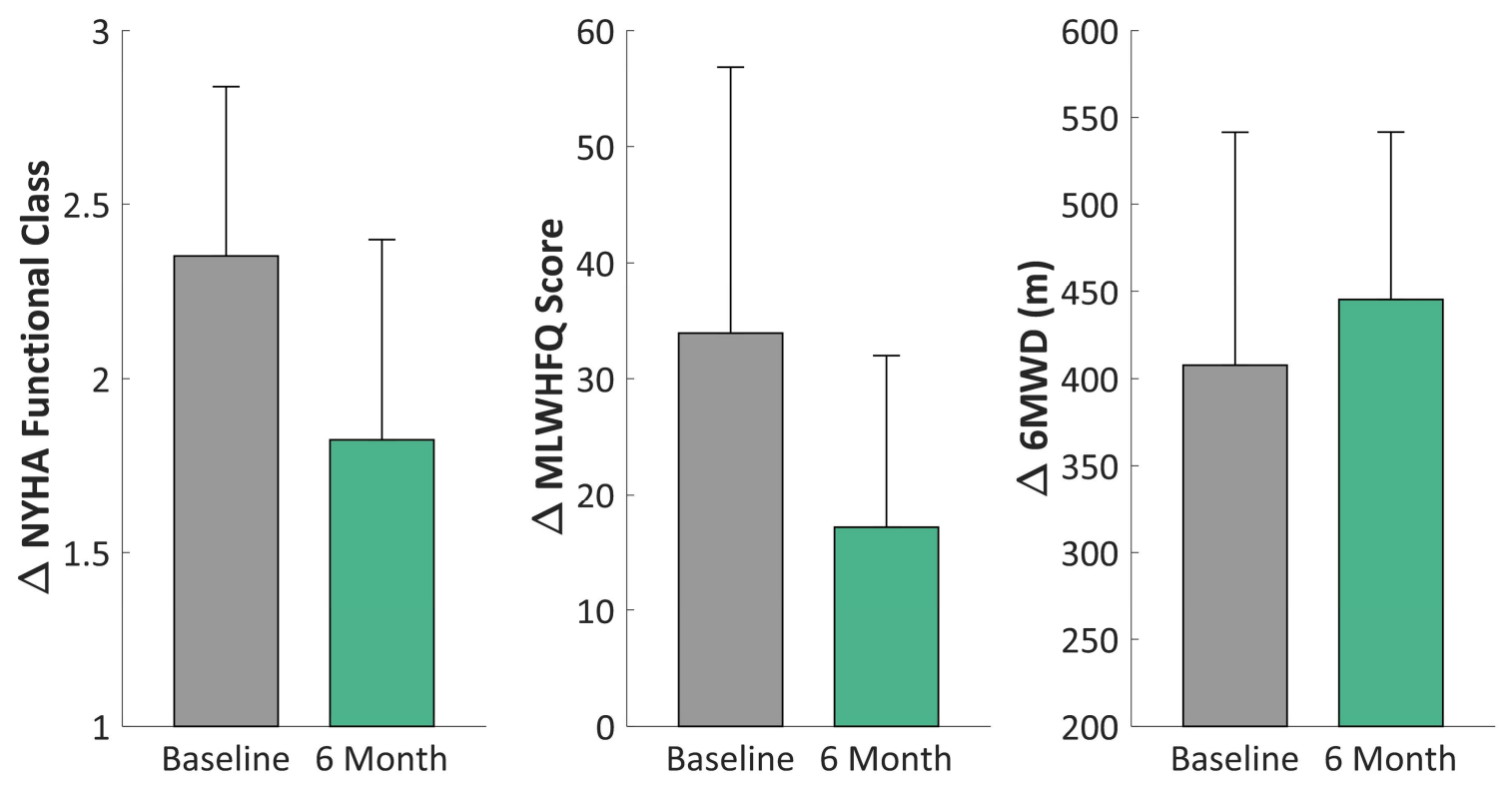